IJMMS 32:2 (2002) 65-71

PII. S0161171202202306

http://ijmms.hindawi.com

(c) Hindawi Publishing Corp.

\title{
ON THE MAXIMUM VALUE FOR ZYGMUND CLASS ON AN INTERVAL
}

\author{
HUANG XINZHONG, OH SANG KWON, and JUN EAK PARK
}

\author{
Received 2 February 2002
}

\begin{abstract}
We prove that if $f(z)$ is a continuous real-valued function on $\mathbb{R}$ with the properties $f(0)=$ $f(1)=0$ and that $\|f\|_{z}=\inf _{x, t}|(f(x+t)-2 f(x)+f(x-t)) / t|$ is finite for all $x, t \in \mathbb{R}$, which is called Zygmund function on $\mathbb{R}$, then $\max _{x \in[0,1]}|f(x)| \leq(11 / 32)\|f\|_{z}$. As an application, we obtain a better estimate for Skedwed Zygmund bound in Zygmund class.
\end{abstract}

2000 Mathematics Subject Classification: 30C62, 30C75.

1. Introduction and the main results. A continuous real-valued function $f(x)$ on $\mathbb{R}$ is said to belong to the class $\Lambda_{*}(\mathbb{R})$ if there exists a constant $C$ such that

$$
|f(x+t)-2 f(x)+f(x-t)| \leq C|t|,
$$

for all $x, t \in \mathbb{R}$. This class introduced by Zygmund [8] is called Zygmund class, and we denote the infimum of the values $C$ in (1.1) by $\|f\|_{z}$. Gardiner and Sullivan [6] proved that by applying the Beurling-Ahlfors extension formula [1] to the function $f(x) \in \Lambda_{*}(\mathbb{R})$, then the Beurling-Ahlfors extension $F_{\mathrm{BA}}=U(x, y)+i V(x, y)$, where

$$
\begin{aligned}
& U(x, y)=\frac{1}{2 y} \int_{x-y}^{x+y} f(t) d t \\
& V(x, y)=\frac{1}{y}\left(\int_{x}^{x+y} f(t) d t-\int_{x-y}^{x} f(t) d t\right),
\end{aligned}
$$

has bounded $\bar{\partial}$-derivative in the upper half plane $H=\{(x, y) \mid y>0\}$, where $\bar{\partial}=$ $\partial_{x}+i \partial_{y}$. On the other hand, from Ahlfors and Bers [2], for any $L_{\infty}$ complex-valued function $\mu(z)$ defined for $z$ in $\mathbb{C}$ there is a curve of quasi-conformal homeomorphisms $f^{t \mu}$ of $\hat{C}$ defined for $|t|<\|\mu\|_{\infty}^{-1}$ such that $f^{t \mu}$ is holomorphic as a function of $t$ and its derivative in $t$ is given by the following formula:

$$
f^{t \mu}=z+t F(z)+O\left(t^{2}\right),
$$

where the constant in $O\left(t^{2}\right)$ is uniform for $z$ in compact sets. If $f^{t \mu}$ is normalized to fix 0,1 , and $\infty$, then $F(x)$ is in the function space $\Lambda_{*}(\mathbb{R})$. The necessary and sufficient condition for a real-valued function $f(x)$ on $\mathbb{R}$ to have an extension $F(z)$ on $H$ with bounded $\bar{\partial}$-derivative is $f(x) \in \Lambda_{*}(\mathbb{R})$, which is proved by Gardiner and Sullivan in [6] also by Reich and Chen in [7]. It is hoped that the knowledge of the special properties of such functions may be applied to the study of quasi-conformal theory.

In order to prove that the Beurling-Ahlfors extension $F_{\mathrm{BA}}$ has bounded $\bar{\partial}$-derivative, Gardiner and Sullivan [6] applied these Zygmund function properties by solving the es- 
timate of $\max \{|f(x)|: 0 \leq x \leq 1\}$ when $f \in \Lambda_{*}(\mathbb{R})$ is normalized by $f(0)=f(1)=0$, in fact, they proved the following theorem.

THEOREM 1.1. Suppose $f(x) \in \Lambda_{*}(\mathbb{R})$, and $f(0)=f(1)=0$. Then,

$$
M=\max \{|f(x)|: 0 \leq x \leq 1\} \leq \frac{1}{2}\|f\|_{z} .
$$

In 1995, Chen and Wei [4] said that the above result could be improved and they showed the following theorem.

THEOREM 1.2. Suppose $f(x) \in \Lambda_{*}(\mathbb{R})$, and $f(0)=f(1)=0$. Then,

$$
M=\max \{|f(x)|: 0 \leq x \leq 1\} \leq \frac{1}{3}\|f\|_{z} .
$$

More recently, in their joint paper, Baladi et al. [3] also used the Skewed Zygmund bound property to estimate the upper and lower bound for some transfer operators. They introduced the Zygmund space $Z$ on $I$, where $I$ denotes a compact interval as the complex vector space of continuous functions $\varphi: I \rightarrow \mathbb{C}$ such that

$$
Z(\varphi)=\sup _{\substack{x \in I \\ t>0: x \pm t \in I}}|Z(\varphi, x, t)|<\infty
$$

where $Z(\varphi, x, t)=(\varphi(x+t)+\varphi(x-t)-2 \varphi(x)) / t$. And they proved the following useful result.

THEOREM 1.3 (Skewed Zygmund bound). For all $\varphi \in Z, x, y \in I$, where I denotes a compact interval, $0<t<1$,

$$
|(1-t) \varphi(x)+t \varphi(y)-\varphi((1-t) x+t y)| \leq \frac{1}{2} Z(\varphi)|x-y| .
$$

In this paper, first we will point out that the proof of the theorem has error, so that Theorem 1.2 is not proved. And then we will prove the following theorem.

THEOREM 1.4. Suppose $f(x) \in \Lambda_{*}(\mathbb{R})$, and $f(0)=f(1)=0$. Then,

$$
M=\max \{|f(x)|: 0 \leq x \leq 1\} \leq \frac{11}{13}\|f\|_{z} .
$$

As an application, we will use our result to obtain a better estimate for the above Skewed Zygmund bound in Section 3.

2. Preliminary results and the proof of Theorem 1.4. We assume $f \in \Lambda_{*}(\mathbb{R})$ and $f(0)=f(1)=0$, and we need the following results due to Chen and Wei [4].

LEMMA 2.1. Let $f \in \Lambda_{*}(\mathbb{R})$, and $\max \{|f(a)|,|f(b)|\} \leq A$. Then,

$$
\begin{aligned}
&\left|f\left(\frac{a+b}{2}\right)\right| \leq A+\frac{b-a}{4}\|f\|_{z}, \\
&\left|f\left(\frac{3 a+b}{4}\right)\right| \leq A+\frac{b-a}{4}\|f\|_{z}, \\
& \max _{x \in[a, b]}|f(x)| \leq A+\frac{b-a}{2}\|f\|_{z} .
\end{aligned}
$$


First, we will point out that there is an error in the proof of Theorem 1.2. Chen and Wei set in [4] that

$$
\left[a_{0}, b_{0}\right]=[0,1], \quad\left[a_{1}, b_{1}\right]=\left[\frac{3 a_{0}+b_{0}}{4}, \frac{a_{0}+b_{0}}{2}\right],
$$

and, by deduction, they set

$$
\left[a_{n}, b_{n}\right]=\left[\frac{3 a_{n-1}+b_{n-1}}{4}, \frac{a_{n-1}+b_{n-1}}{2}\right] .
$$

As Chen and Wei used in [4], they denoted by $\Lambda_{n}$ the Zygmund class on the interval $\left[a_{n}, b_{n}\right], n=0,1, \ldots$ with $\max \left\{\left|f\left(a_{n}\right)\right|,\left|f\left(b_{n}\right)\right|\right\} \leq A_{n}$ and $\|f\|_{z} \leq B$, then they derived that

$$
\sup _{x \in\left[a_{0}, b_{0}\right], f \in \Lambda_{0}}|f(x)| \leq \sup _{x \in\left[a_{1}, b_{1}\right], f \in \Lambda_{1}}|f(x)| \leq \sup _{x \in\left[a_{2}, b_{2}\right], f \in \Lambda_{2}}|f(x)| .
$$

Contradicting to Chen and Wei [4], we say that the method used to obtain (2.5) does not generally hold, and the formulas of (2.5) can only be true in the following

$$
\sup _{x \in\left[a_{n}, b_{n}\right], f \in \Lambda_{n-1}}|f(x)| \leq \sup _{x \in\left[a_{n}, b_{n}\right], f \in \Lambda_{n}}|f(x)| .
$$

For if were true, by the same method used by Chen and Wei [4], we set that

$$
\left[a_{1}, b_{1}\right]=\left[0, \frac{1}{8}\right]
$$

let $\Lambda_{1}$ be the Zygmund class on the interval $\left[a_{1}, b_{1}\right]$ with $\max \left\{\left|f\left(a_{1}\right)\right|,\left|f\left(b_{1}\right)\right|\right\} \leq A_{1}$ and $\|f\|_{z} \leq B$, then

$$
\sup _{x \in\left[a_{0}, b_{0}\right], f \in \Lambda_{0}}|f(x)| \leq \sup _{x \in\left[a_{1}, b_{1}\right], f \in \Lambda_{1}}|f(x)| .
$$

On the other hand, by the definition of (1.1), if we set $x=t=1 / 8$, then $|f(1 / 8)| \leq$ $3 B / 16$, we have $\max \left\{\left|f\left(a_{1}\right)\right|,\left|f\left(b_{1}\right)\right|\right\} \leq 3 B / 16$, and $\|f\|_{z} \leq B$, by (2.2), then

$$
\max _{x \in[0,1 / 8]}|f(x)| \leq \frac{3 B}{16}+\frac{B}{16}=\frac{B}{4} .
$$

Hence by (2.8), we can derive that

$$
\sup _{x \in[0,1], f \in \Lambda_{0}}|f(x)| \leq \sup _{x \in[0,1 / 8], f \in \Lambda_{1}}|f(x)| \leq \max _{x \in[0,1 / 8]}|f(x)| \leq \frac{B}{4} .
$$

The following example is used in [4].

EXAMPLE 2.2. A piecewise linear function $f_{*}(x) \in \Lambda_{*}(\mathbb{R})$ with $\left\|f_{*}\right\|=1$ is defined as follows (also see [4]). 
We choose that $f(x)$ equals zero when $x<0$ and $x>1$, the dividing points in $[0,1]$ and the values of $f_{*}$ at the dividing points are listed as follows:

$$
f_{*}(x)= \begin{cases}0, & x=0,1, \\ \frac{1}{4}, & x=\frac{1}{4}, \frac{1}{2}, \frac{3}{4} \\ \frac{5}{16}, & x=\frac{3}{8}, \frac{5}{8} .\end{cases}
$$

By the above example and (2.10), we have

$$
\max _{x \in[0,1], f \in \Lambda_{0}}|f(x)| \leq \frac{1}{4}|| f_{*} \|=\frac{1}{4}
$$

but, $f(3 / 8)=5 / 16>1 / 4$, which is a contradiction.

Thus the inequalities in (2.5) do not hold in general.

Proof of TheOrem 1.4. Suppose $f \in \Lambda_{*}(\mathbb{R})$, and $f(0)=f(1)=0$, from Lemma 2.1 , for any $B \geq\|f\|_{z}$, we see that if we choose $x=t=1 / 2$, then, by $(1.1),|f(1 / 2)| \leq$ $B / 4$, and if $x=t=1 / 4$, then $|f(1 / 2)-2 f(1 / 4)| \leq B / 4$, and $|f(1 / 4)| \leq B / 4$, also if we choose $x=t=1 / 8$, we have $|f(1 / 4)-2 f(1 / 8)| \leq B / 8$, and $|f(1 / 8)| \leq 3 B / 16$.

By (2.2), we obtain the estimate in the interval of $[0,1 / 8]$

$$
\max _{x \in[0,1 / 8]}|f(x)| \leq \frac{3 B}{16}+\frac{B}{16}=\frac{B}{4}
$$

while in the interval of $[1 / 8,1 / 4]$, because of $\max \{|f(1 / 8)|,|f(1 / 4)|\} \leq B / 4$, the same way can be used to get that

$$
\max _{x \in[1 / 8,1 / 4]}|f(x)| \leq \frac{B}{4}+\frac{B}{16}=\frac{5 B}{16} .
$$

Again, if we choose $x=3 / 8$ and $t=1 / 8$, by (1.1), then

$$
\left|f\left(\frac{1}{2}\right)-2 f\left(\frac{3}{8}\right)+f\left(\frac{1}{4}\right)\right| \leq \frac{B}{8}, \quad\left|f\left(\frac{3}{8}\right)\right| \leq \frac{5 B}{16},
$$

and if we choose $x=1 / 4, t=1 / 2$, from (1.1), we obtain

$$
\left|f\left(\frac{3}{8}\right)-2 f\left(\frac{5}{16}\right)+f\left(\frac{1}{4}\right)\right| \leq \frac{B}{16}, \quad\left|f\left(\frac{5}{16}\right)\right| \leq \frac{5 B}{16}
$$

Thus, $\max \{|f(1 / 4)|,|f(5 / 16)|\} \leq 5 B / 16$, we can derive by (2.2) in Lemma 2.1 that

$$
\max _{x \in[1 / 4,5 / 16]}|f(x)| \leq \frac{5 B}{16}+\frac{B}{32}=\frac{11 B}{32} .
$$


For the interval of $[5 / 16,3 / 8]$, we also get, by (2.2), that

$$
\max _{x \in[5 / 16,3 / 8]}|f(x)| \leq \frac{11 B}{32} .
$$

Let $x=7 / 16$, and $t=1 / 16$, by (1.1), we derive that

$$
\left|f\left(\frac{1}{2}\right)-2 f\left(\frac{7}{16}\right)+f\left(\frac{3}{8}\right)\right| \leq \frac{B}{16}, \quad\left|f\left(\frac{7}{16}\right)\right| \leq \frac{5 B}{16}\|f\|_{z} .
$$

Thus, in the interval of $[3 / 8,7 / 16]$, we obtain from $\max \{|f(3 / 8)|,|f(7 / 16)|\} \leq 5 B / 16$, and by (2.2), that

$$
\max _{x \in[3 / 8,7 / 16]}|f(x)| \leq \frac{11 B}{32} .
$$

Also since we have $|f(7 / 16)| \leq 5 B / 16$ and $|f(1 / 2)| \leq B / 4$, so we have the estimate in the interval of $[7 / 16,1 / 2]$, that is

$$
\max _{x \in[7 / 16,1 / 2]}|f(x)| \leq \frac{11 B}{32} .
$$

Combining with the above estimates from (2.13), (2.14), (2.17), (2.18), (2.20), and (2.21), we obtain that

$$
\max _{x \in[0,1 / 2]}|f(x)| \leq \frac{11 B}{32} .
$$

By the fact that if $f(x) \in \Lambda_{*}(\mathbb{R})$, then $f_{*}(x)=(1 / a) f(a x+b)+c x+d \in \Lambda_{*}(\mathbb{R})$, and $\left\|f_{*}\right\|_{z}=\|f\|_{z}$ for any real constants $a \neq 0, b, c$ and $d$, which is called linear-invariant property, if $f \in \Lambda_{0}$, let $F(x)=-f(1-x) \in \Lambda_{0}$, by (2.22), then we obtain that

$$
\max _{x \in[0,1 / 2]}|F(x)|=\max _{x \in[1 / 2,1]}|f(x)| \leq \frac{11 B}{32} .
$$

The results we obtain hold for any $B \geq\|f\|_{z}$, hence we have proved that

$$
\max _{x \in[0,1]}|f(x)| \leq \frac{11}{32}\|f\|_{z} .
$$

The proof of Theorem 1.4 is finished.

3. Application to estimate Skewed Zygmund bound. We will use our Theorem 1.4 to improve Skewed Zygmund bound due to Baladi et al. [3]. Suppose $I$ is a compact interval and the Zygmund space $Z$ on $I$ as the complex vector space of continuous functions $\varphi: I \rightarrow \mathbb{C}$ such that

$$
Z(\varphi)=\sup _{\substack{x \in I \\ t>0: x \pm t \in I}}|Z(\varphi, x, t)|<\infty,
$$

where $Z(\varphi, x, t)=(\varphi(x+t)+\varphi(x-t)-2 \varphi(x)) / t$. 
The vector space $Z$ becomes a Banach space when it is endowed with the norm $\|\varphi\|=\max \left(\sup _{I}|\varphi|, Z(\varphi)\right)$, and it has close relation with the Banach space $\Lambda^{\alpha}$ of $\alpha$-Hölder functions, that is, functions $\varphi: I \rightarrow \mathbb{C}$ satisfying

$$
|\varphi|_{\alpha}=\sup _{x \neq y \in I} \frac{|\varphi(x)-\varphi(y)|}{|x-y|^{\alpha}}<\infty, \quad 0<\alpha \leq 1,
$$

with the norm $\|\varphi\|_{\alpha}=\max \left(\sup _{I}|\varphi|,|\varphi|_{\alpha}\right)$. We know that $Z \varsubsetneqq \Lambda^{\alpha}$ for $0<\alpha<1$ and $\Lambda^{1} \varsubsetneqq Z$. (See $[3,5]$.) Our next result will be stated in the following theorem.

THEOREM 3.1 (Skewed Zygmund bound). For all $\varphi \in Z, x, y \in I$, where I denotes a compact interval, $0<t<1$,

$$
|(1-t) \varphi(x)+t \varphi(y)-\varphi((1-t) x+t y)| \leq \frac{11}{32} Z(\varphi)|x-y| .
$$

Proof OF TheOrem 3.1. Suppose a given function $\varphi(x)$ satisfying the conditions in Theorem 3.1, for any $x, y \in I$ we define a function in $[0,1]$ as follows:

$$
F(t)=\frac{1}{y-x} \varphi(t(y-x)+x)+\frac{\varphi(x)-\varphi(y)}{y-x} t-\frac{\varphi(x)}{y-x}
$$

then we have a continuous function $F(t)$ in $[0,1]$ satisfying

$$
\begin{aligned}
& F(0)=\frac{1}{y-x} \varphi(x)-\frac{1}{y-x} \varphi(x)=0, \\
& F(1)=\frac{\varphi(y)}{y-x}+\frac{\varphi(x)-\varphi(y)}{y-x}-\frac{\varphi(x)}{y-x}=0 .
\end{aligned}
$$

By the linear-invariant property, we obtain that $F(t) \in \Lambda_{0}$, and

$$
Z(F)=Z(\varphi)
$$

By Theorem 1.4, we have

$$
\max _{t \in[0,1]}|F(t)| \leq \frac{11}{32} Z(\varphi) .
$$

However, we see that

$$
\begin{aligned}
|F(t)| & =\left|\frac{\varphi(t(y-x)+x)}{y-x}+\frac{t \varphi(x)-t \varphi(y)-\varphi(x)}{y-x}\right| \\
& =\left|\frac{\varphi((1-t) x+t y)+(t-1) \varphi(x)-t \varphi(y)}{y-x}\right| \\
& =\left|\frac{((1-t) \varphi(x)+t \varphi(y))-\varphi((1-t) x+t y)}{y-x}\right| \\
& \leq \frac{11}{32} Z(\varphi) .
\end{aligned}
$$


Hence, we have by (3.7) that

$$
|\varphi((1-t) x+t y)-((1-t) \varphi(x)+t \varphi(y))| \leq \frac{11}{32} Z(\varphi)|x-y| .
$$

The proof of Theorem 3.1 is finished.

REMARK 3.2. There are also some useful applications of Theorems 1.4 and 3.1, for example, they can be used for the estimate of the upper and lower bound for some transfer operators introduced in [4], we omit it here.

ACKNOWLEDGment. The first author expresses his thanks to the Department of Mathematics, Kyungsung University, for the support received during his stay at Kyungsung University in Korea.

\section{REFERENCES}

[1] L. V. Ahlfors, Lectures on Quasiconformal Mappings, Van Nostrand Mathematical Studies, no. 10, D. Van Nostrand, New York, 1966.

[2] L. V. Ahlfors and L. Bers, Riemann's mapping theorem for variable metrics, Ann. of Math. (2) 72 (1960), 385-404.

[3] V. Baladi, Y.-P. Jiang, and O. E. Lanford III, Transfer operators acting on Zygmund functions, Trans. Amer. Math. Soc. 348 (1996), no. 4, 1599-1615.

[4] J. Chen and H. Wei, On some constants of quasiconformal deformation and Zygmund class, Chinese Ann. Math. Ser. B 16 (1995), no. 3, 325-330.

[5] W. de Melo and S. van Strien, One-Dimensional Dynamics, Ergebnisse der Mathematik und ihrer Grenzgebiete (3), vol. 25, Springer-Verlag, Berlin, 1993.

[6] F. P. Gardiner and D. P. Sullivan, Symmetric structures on a closed curve, Amer. J. Math. 114 (1992), no. 4, 683-736.

[7] E. Reich and J. Chen, Extensions with bounded $\bar{\partial}$-derivative, Ann. Acad. Sci. Fenn. Ser. A I Math. 16 (1991), no. 2, 377-389.

[8] A. Zygmund, Smooth functions, Duke Math. J. 12 (1945), 47-76.

Huang Xinzhong: Department of Applied Mathematics, Huaqiao national UniverSITY, QUANZHOU, FUJIAN 362011, CHINA

E-mail address: huangxz@nqu.edu.cn

OH SANG Kwon: Department of Mathematics, Kyungsung University, Pusan 608-736, KOREA

E-mail address: oskwon@star. kyungsung.ac.kr

Jun Eak Park: Department of Mathematics, Kyungsung University, Pusan 608-736, KOREA 


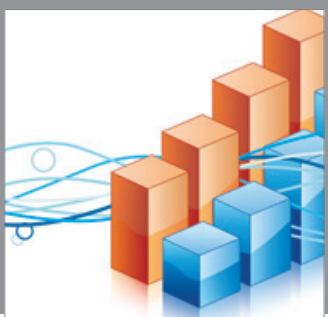

Advances in

Operations Research

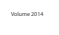

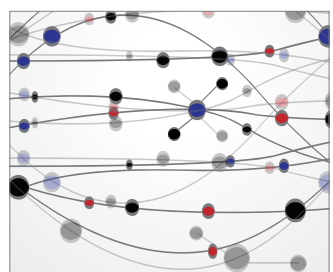

\section{The Scientific} World Journal
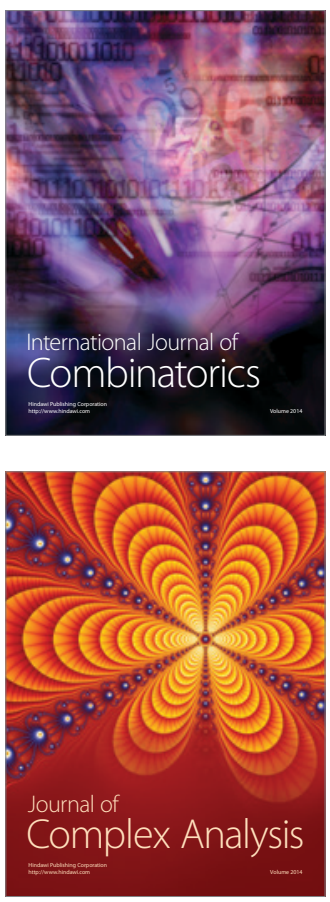

International Journal of

Mathematics and

Mathematical

Sciences
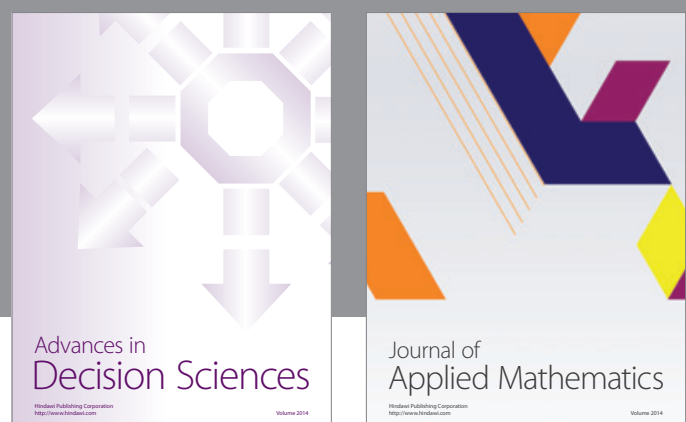

Journal of

Applied Mathematics
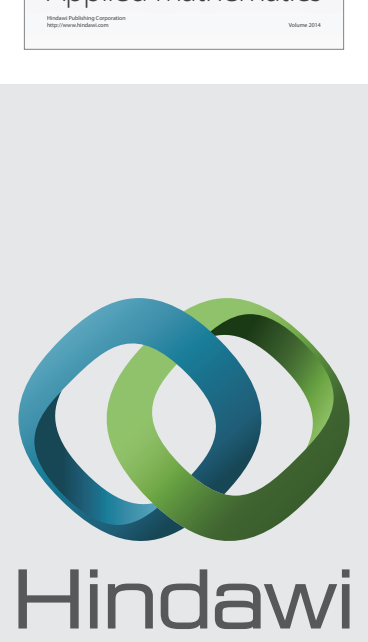

Submit your manuscripts at http://www.hindawi.com
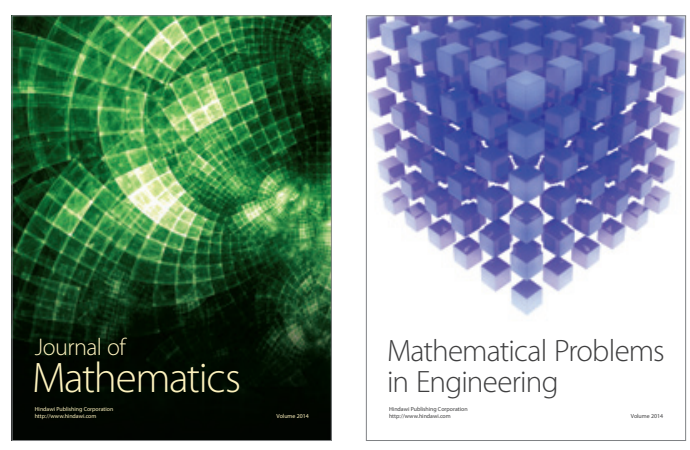

Mathematical Problems in Engineering
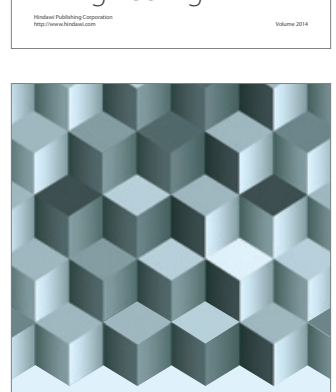

Journal of

Function Spaces
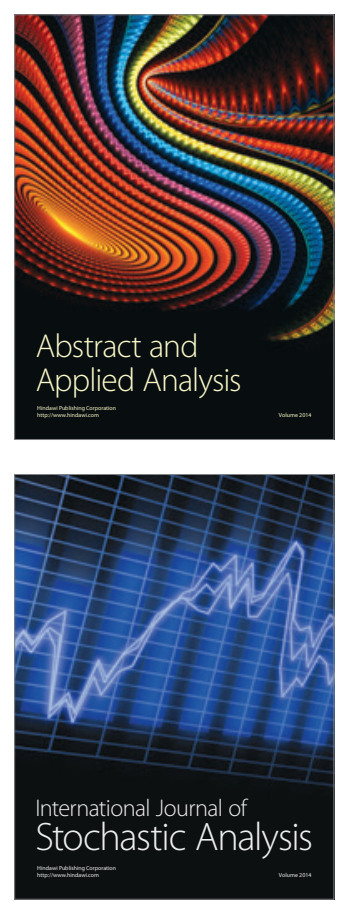

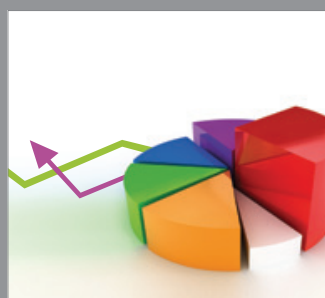

ournal of

Probability and Statistics

Promensencen
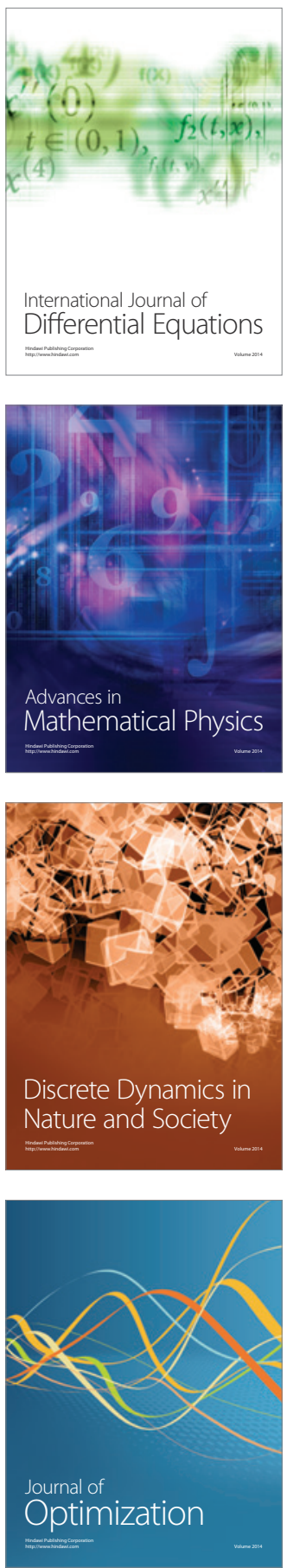